\title{
Rex factus est uxorius: Weibliche und männliche Herrschaftsrollen in Outremer
}

\author{
Goridis, Philippe
}

DOI: https://doi.org/10.1515/mial-2016-0003

Posted at the Zurich Open Repository and Archive, University of Zurich ZORA URL: https://doi.org/10.5167/uzh-124522

Journal Article

Published Version

Originally published at:

Goridis, Philippe (2016). Rex factus est uxorius: Weibliche und männliche Herrschaftsrollen in Outremer. Das Mittelalter. Perspektiven mediävistischer Forschung, 21(1):22-39.

DOI: https://doi.org/10.1515/mial-2016-0003 
Philippe Goridis*

\section{Rex factus est uxorius. Weibliche und männliche Herrschaftsrollen in Outremer}

DOI 10.1515/mial-2016-0003

Abstract: By the 1130s at the latest, women played an important role in the politics of Outremer. This was due to the belligerent crusader environment which took its toll on male crusaders. Although the proportion of queens regnant was striking, the general situation of ruling women in the Holy Land bears some resemblance to Western European regions and should thus not be seen as exceptional. The rule of women was initially looked on with scepticism and was only considered if no other (male) candidates were available. Women who exercised governmental powers were normally seen as regents by kinship for minor heirs rather than as rulers in their own right. Therefore, they were usually replaced by a male regent as soon as possible. And if they were able to act as independent rulers, they were often described in masculine terms. However, women were endowed with one important political right: the ideas of female succession to property were well established in the 12th century and female legal and ruling rights were further developed until the mid-13th century. These processes led to an enlargement of the potential range of activity of women who could assume diverse ruling functions in the 13th century. By then at the latest, female and male ruling roles and activities complemented one another and became - to a certain degree - mutually dependent. However, this did not mean that every woman with a right to rule acted as a ruler. In this regard, men still dominated the political landscape of Outremer.

Keywords: Crusader Principalities, Outremer, Queenship, Kingship, Gender Roles

Rex autem ab ea die ita factus est uxorius [...] quod nec in causis levibus, absque ejus conscientia attemptaret aliquatenus procedere. ${ }^{1}$ Der König von Jerusalem sei nach einem verlorenen Machtkampf zum Sklaven seiner Gemahlin geworden und

1 Wilhelm von Tyrus, Chronicon. Hrsg. v. Robert Huygens (Corpus Christianorum Continuatio Mediaevalis 63, 63A). Turnhout 1986, hier XIV, 18, S. 656. Zur Übersetzung ins Englische vgl. William of Tyre, A History of Deeds Done Beyond the Sea. Übers. v. Emily Atwater Babcock u. August C. Krey. New York 1943.

*Kontakt: Dr. Philippe Goridis, Historisches Seminar, Universität Zürich, Karl Schmid-Strasse 4, CH-8006 Zürich, E-Mail: philippe.goridis@hist.uzh.ch 
habe auch in den einfachsten Angelegenheiten nicht mehr gewagt, ohne ihre Zustimmung zu entscheiden ${ }^{2}$ - mit diesen Worten beschreibt Wilhelm von Tyrus, der große Chronist der Kreuzzugszeit, eine einschneidende Veränderung im Verhältnis zwischen König Fulko und seiner Frau, der Erbkönigin Melisende von Jerusalem. Er markiert damit den Beginn einer Entwicklung, die das konstitutionelle Gefüge der christlichen Reiche im Heiligen Land veränderte und den Umgang von Herrscherinnen und Herrschern miteinander prägen sollte. Das Zitat impliziert einen Rollentausch zwischen rex und regina. Wilhelm deutet damit zumindest eine Aufweichung des traditionellen genderdifferenzierten Rollenschemas an: Der König ,verweiblicht‘; er gibt Kompetenzen ab, während seine Frau auf der politischen Entscheidungsebene massiv an Bedeutung gewinnt.

Tatsächlich kam den Herrscherinnen in den Kreuzfahrerherrschaften im europäischen Vergleich große Bedeutung zu. Immer wieder standen sie als Erbtöchter wenigstens nominell an der Spitze ihrer jeweiligen Reiche und regierten diese in der Regel zusammen mit ihren Ehemännern - Konstanze in Antiochia (1136-1163) und Melisende (1131-1152), Sibylle (1186-1190), Isabella I. (1192-1205), Maria la Marquise (1210-1212) und Isabella II. (1225-1228) in Jerusalem. ${ }^{3}$ Entsprechende Aufmerksamkeit hat in den letzten Jahrzehnten die Erforschung besonders des Jerusalemer Königinnentums erfahren. ${ }^{4}$ Dennoch ist der Kenntnisstand über das Königtum und die mit ihm verknüpften Institutionen ungleich größer; nur vereinzelt geriet das Verhältnis zwischen Herrscherin und Herrscher und das darauf aufbauende Rollenverständnis von Mann und Frau in den Fokus des Interesses. ${ }^{5}$

2 Zur Vorgeschichte vgl. weiter unten sowie detailliert Alan V. Murray, Baldwin II and His Nobles. Baronial Factionalism and Dissent in the Kingdom of Jerusalem, 1118-1134. In: Nottingham Medieval Studies 38 (1994), S. 60-85, hier S. 79-85.

3 Alan V. Murray, Women in the Royal Succession of the Latin Kingdom of Jerusalem (10991291). In: Claudia Zey (Hg.), Mächtige Frauen? Königinnen und Fürstinnen im europäischen Mittelalter (11.-14. Jahrhundert) (Vorträge und Forschungen 81). Ostfildern 2015, S. 131-162, hier S. 136 errechnet für die rund zweihundertjährige Existenz der Kreuzfahrerherrschaften einen Anteil weiblicher Thronfolgen von 31\%, während er für den lateinischen Westen in einem Zeitraum von fünfhundert Jahren einen Anteil weiblicher Herrschaftssukzession von deutlich unter $12 \%$ annimmt.

4 Exemplarisch: Murray (Anm. 3); Sarah Lambert, Queen or Consort. Rulership and Politics in the Latin East, 1118-1228. In: Anne Duggan (Hg.), Queens and Queenship in Medieval Europe. Proceedings of a Conference Held at King's College London April 1995. Woodbridge 1997, S. 153-169; Hans Eberhard Mayer, Studies in the History of Queen Melisende of Jerusalem. In: Dumbarton Oaks Papers 26 (1972), S. 93-182; für weitere Literatur vgl. Philippe Goridis, Gefährten, Regenten, Witwer. Männliche Herrschaft im Heiligen Land der Erbköniginnen. In: Zey (Anm. 3), S. 163-197, bes. S. 163 , Anm. 1 .

5 Für Arbeiten, die weibliches und männliches Herrschaftshandeln eher im Verbund betrachten, vgl. Goridis (Anm. 4), dort auch weitere Literatur. 
Ziel dieses Beitrags ist es, dieses Rollenverständnis sowie die damit einhergehenden Handlungsräume explizit in den Blick zu nehmen und mit der konstitutionellen Entwicklung Outremers abzugleichen. Deshalb ist im Folgenden erstens nach den Rollenzuschreibungen aus Sicht der Quellen zu fragen, zweitens und drittens eine Einordnung in die politischen Zusammenhänge und Veränderungen des 12. und des 13. Jahrhunderts vorzunehmen, ehe viertens ein weiterführendes Fazit den Beitrag beschließt. Als Quellengrundlage dienen die erzählende, die diplomatische sowie die rechtliche Überlieferung aus den Kreuzfahrerherrschaften, die vergleichend in gegenseitiger Ergänzung $\mathrm{zu}$ betrachten sind.

\section{Frauen- und Männerrollen in Outremer}

Einer der wichtigsten und einprägsamsten Informationsträger zum geschlechtsspezifischen Herrschaftshandeln ist die eingangs zitierte Chronik Wilhelms von Tyrus. Dank ihres Detailreichtums und ihrer Bedeutung für die Geschichte Outremers bildet sie eine wertvolle Basis für grundlegende Aussagen. Wilhelm ordnete seine Erzählung in dreiundzwanzig Bücher, die sich durchgehend an der Abfolge der männlichen Herrscher orientieren - selbst wenn sie sich in die königliche Dynastie lediglich eingeheiratet hatten und sich ihr Regierungsanspruch ausschließlich über die Erbköniginnen herleitete. Wilhelm erzählt also in erster Linie eine männliche Herrschaftsgeschichte, ungeachtet der beträchtlichen Rolle, die Frauen mitunter darin spielten.

Dieser Befund lässt sich weitgehend auch auf die anderen narrativen Quellen ausdehnen, die unser Bild von der Geschichte der Kreuzfahrerherrschaften prägen. ${ }^{6}$ Dadurch wird die Bedeutung von Frauen für das Herrschaftsgefüge des Heiligen Landes verschleiert. Als Ehefrauen übernehmen sie in den Chroniken die Funktion von Transmittern familiärer Verbindungen, als Erbinnen vermitteln und sichern sie Herrschaftsansprüche und als Mütter sorgen sie für ihren Nachwuchs. Die Chronistik stellt sie ihren Männern meist beiläufig zur Seite, ohne ihnen eine aktive (politische) Funktion zuzugestehen.

Zeitgenössische Berichterstatter vor allem des 12. Jahrhunderts betrachteten die Eigenständigkeit von Herrscherinnen häufig als Anomalie und damit als Bruch der männlich dominierten Rahmenerzählung. Besonders gut lässt sich dies

6 Zur Quellenlage vgl. Philippe Goridis, Gefangen im Heiligen Land. Verarbeitung und Bewältigung christlicher Gefangenschaft zur Zeit der Kreuzzüge (Vorträge und Forschungen, Sonderband 57). Ostfildern 2015, S. 47-52. 
erneut am Beispiel Wilhelms von Tyrus aufzeigen. Wilhelm erkannte prinzipiell das Recht von Frauen an, Besitzungen und Herrschaftstitel zu erben oder Regentschaften zu führen. ${ }^{7}$ Wenn sie selbst aber Herrschaft ausübten, beurteilte er sie in der Regel äußerst skeptisch, auch wenn er durchaus ihre Tugenden und Verdienste wie adelige Herkunft, Züchtigkeit, Anstand, Gottesfürchtigkeit und das redliche Bemühen um gutes Regieren bemerkte. ${ }^{8}$ Als im Jahr 1149 Fürst Raimund von Antiochia fiel und wenig später Graf Joscelin II. von Edessa in muslimische Gefangenschaft geriet, sodass deren Reiche unter die Kontrolle der zurückgebliebenen Ehefrauen kamen, kommentierte er die Lage abschätzig: Sic igitur peccatis nostris exigentibus utraque regio, melioribus destituta consiliis vix in se substinens, femineo regebatur imperio. ${ }^{9}$

Frauenherrschaft, hier sogar als Strafe für Sünden gesehen, konnte in Wilhelms Augen nur dann funktionieren, wenn den Erbherrinnen geeignete (Ehe-) Männer zur Seite standen, die aktiv regierten - eine Vorstellung, die mit der Struktur seiner Chronik korrespondiert. Alleinherrschende Frauen zeichnete er als schwach oder intrigant; deshalb kritisierte er sie, wenn sie nicht zu einer Heirat zu bewegen waren - denn ihnen fehle, so Wilhelm, die Fähigkeit, das Amt eines Fürsten auszufüllen (principis fungere officio). ${ }^{10}$ So bezeichnete er Alice von Antiochia, die Witwe Bohemunds II., explizit als böse, als diese zum wiederholten Mal nach der Herrschaft über das Fürstentum strebte. ${ }^{11}$ Und als sich Konstanze von Antiochia nach dem Tod ihres ersten Ehemannes schließlich weigerte, einen neuen Gemahl zu nehmen, warf er ihr vor, ihr frivoles Leben über die Bedürfnisse ihres Volkes zu stellen. ${ }^{12}$

Nur in einem Fall, nämlich jenem der Königin Melisende von Jerusalem, wich Wilhelm von diesem Muster ab. In ihr sah der Chronist nicht nur eine weise und gütige Frau, sondern auch eine tatkräftige Herrscherin, die keinen Vergleich mit anderen Fürsten oder gar ihren männlichen Amtsvorgängern zu scheuen brauchte. Ihr Herrschaftshandeln habe die Kräfte einer Frau weit überstiegen. Analog zu Fulkos Verweiblichung gestand er Melisende eine Vermännlichung zu, die sie seiner prinzipiellen Kritik an ihren Geschlechtsgenossinnen enthob. ${ }^{13}$

7 Vgl. etwa Wilhelm von Tyrus (Anm. 1), XIV, 4, S. 635; XV, 27, S. 711; XVII, 13, S. 777; XXI, 4,

S. 964; XXI, 17, S. 986; XXII, 10, S. 1019.

8 Ebd., XIV, 3, S. 635; XVII, 11, S. 775.

9 Ebd., XVII, 11, S. 775.

10 Wilhelm von Tyrus (Anm. 1), XVII, 10, S. 773.

11 Ebd., XIII, 27, S. 624; XIV, 4, S. 635 f.

12 Ebd., XVII, 18, S. $785 \mathrm{f}$.

13 Ebd., XV, 27, S. 711; XVI, 3, S. 717; XVII, 1, S. 761; XVII, 13, S. 777; XVIII, 27, S. 850 f. Ähnlich äußert sich Wilhelm ebd., XVII, 11, S. 775 über die Regierung der Beatrix in Edessa nach der Gefangennahme ihres Mannes Joscelin II. 
Über die Gründe für Wilhelms Affinität zu Melisende kann nur spekuliert werden. ${ }^{14}$ In jedem Fall ist bemerkenswert, dass Wilhelm ein positives Herrscherinnenbild offenbar nur mit männlichen Herrscherattributen zu zeichnen in der Lage war und zudem betonte, dass der Königin der Konstabler Manasses von Hierges als tatkräftiger Mann zur Seite gestanden habe. ${ }^{15}$ Die demonstrative Hervorhebung männlicher Berater bei Herrscherinnen eigenen Rechts ist bemerkenswert, zumal sich Vergleichbares im Falle männlicher Potentaten, die sich selbstverständlich auch auf Amtsträger stützten, selten feststellen lässt. Offenbar galt Wilhelm die Präsenz von Männern für das Funktionieren einer Regierung als unerlässlich.

Zeugnisse anderer Zeitgenossen gehen in eine ähnliche Richtung. Bernhard von Clairvaux sprach Melisende nach dem Tod ihres Mannes brieflich zwar Mut $\mathrm{zu}$, doch treten in seinem Schreiben auch deutliche Vorbehalte zutage. Schließlich riet er der Königin ausdrücklich zur Virilisierung, um - die Schwächen einer Frau überwindend - königlich männliche Taten auszuführen, damit niemand frage: Ubi est rex Ierosolymorum ${ }^{16}$ Der hier geforderte Geschlechtswechsel verweist auf die Schwierigkeiten der zeitgenössischen Beobachter, ihr männlich konnotiertes Herrscherbild mit dem erfolgreichen Regieren von Frauen in Einklang zu bringen.

Ein ähnlicher Umdeutungsprozess betrifft Männer, die - aus welchen Gründen auch immer - als nicht herrschaftsbefähigt oder zumindest als nicht voll herrschaftsbefähigt angesehen wurden. Bestes Beispiel dafür ist die Schilderung Wilhelms zu dem sich wandelnden Machtverhältnis zwischen Melisende und Fulko. Ähnliches gilt für Humfried IV. von Toron, den ersten Ehemann der Thron-

14 Vgl. Murray (Anm. 3), S. 143.

15 Wilhelm von Tyrus (Anm. 1), XVII, 13, S. 777 f. Murray (Anm. 3), S. 143 bezeichnet Manasses gar als „substitute husband“.

16 Sancti Bernardi Opera, Bd. 8. Hrsg. v. Jean LeClercq u. Henri Rochais. Rom 1977, Nr. 354: Mortuo rege viro tuo, et parvulo rege adhuc minus idoneo ad portanda negotia regni et ad prosequendum regis officium, oculi omnium in te respiciunt et in te solam universa regni moles inclinata recumbit. Opus est ut manum tuam mittas ad fortia et in muliere exhibeas virum, agens ea quae agenda sunt in spiritu consilii et fortitudinis. Ita prudenter et moderate oportet te cuncta disponere, ut omnes, qui te viderint, ex operibus regem te potius quam reginam existiment, ne forte dicant in gentibus: „Ubi est rex Ierosolymorum?“ „Sed non sum,“ inquies, „ad ista sufficiens. Magna enim haec sunt; supra vires meas, et supra scientiam meam. Opera haec opera sunt viri: ego autem mulier sum, corpore debilis, mobilis corde, nec provida consilio, nec assueta negotiis." Scio, filia, scio, quia magna sunt haec; sed et hoc scio quia, etsi mirabiles elationes maris, mirabilis in altis dominus. Magna sunt haec, sed magnus dominus noster et magna virtus eius. Für die Übersetzung vgl. Joan Ferrante, A letter from Bernard of Clairvaux, abbot (1143-44); http://epistolae.ccnmtl.columbia. edu/letter/246.html (Zugriff: 11.1.2015). 
erbin Isabella I. von Jerusalem. Die Chronistik der frühen 1190er-Jahre hat seine Trennung von Isabella unter anderem damit begründet, dass er ohnehin keine Aussicht gehabt habe, als König zu regieren, denn er sei mehr Frau als Mann freundlich, stotternd, schwachen Herzens und weiblich erzogen (norreture de feme). ${ }^{17}$ Diesem Porträt von Unfähigkeit stehen, analog zu den positiv konnotierten Virilisierungen bei Frauen, allerdings durchaus wohlgemeinte Effeminationen von Männern entgegen: So beschrieb Radulph von Caen Tankred, den Regenten von Antiochia, als eine Löwin auf Beutezug, die ihre Jungen zurücklasse, und Wilhelm von Tyrus schilderte König Fulko als Heilige Martha, die sich aufopfernd um ihr Volk kümmere. ${ }^{18}$

Die diplomatische Überlieferung der Kreuzfahrerherrschaften, über die nach wie vor Reinhold Röhrichts ,Regesta regni Hierosolymitani‘ einen hervorragenden Überblick bieten, bestätigt dieses Bild der erzählenden Quellen. Es sticht sofort ins Auge, dass Frauen nur in den wenigsten Fällen eigene Urkunden ausstellten und selbstständig über Besitzungen und Privilegien verfügten. ${ }^{19}$ Die meisten der erhaltenen Diplome mit weiblicher Beteiligung beinhalten lediglich die Zustimmung der Frau zu einem Rechtsgeschäft, während Ausstellungen in beider Namen weit seltener anzutreffen sind. Gerade die recht häufige Konsensformel zeigt, dass dem Wort der Gemahlinnen durchaus Gewicht beigemessen wurde, etwa um ein Rechtsgeschäft breiter abzustützen und zu gewährleisten, dass die Rechtslage auch nach dem Tod des Ausstellers gesichert war. Dieser Praxis folgte man vorwiegend bei jenen Rechtsgeschäften, die zwingend der Ratifikation durch die Gattin bedurften, also wenn ihr die verfügten Besitzrechte oblagen und der Aussteller nur wegen der Eheverbindung darüber bestimmen

17 L'Estoire de Eracles Empereur et la conqueste de la terre d'Outremer (Recueil des Historiens des Croisades, Historiens Occidentaux 2). Paris 1859, S. 1-481, hier XXV, 11, S. 153; Itinerarium peregrinorum. Eine zeitgenössische englische Chronik zum dritten Kreuzzug in ursprünglicher Gestalt. Hrsg. v. Hans Eberhard Mayer (Schriften der MGH 18). Stuttgart 1962, hier I, 63, S. 352354. Ebd. wird Humfrieds weibliche Seite mit Vergil weiter kommentiert: Dum dubitat natura marem faceretve puellam, natus es, o pulcher, pene puella puer. [...] [N] am varium et mutabile semper femina, [und weiter in eigenen Worten:] cui et sexus lubricus et mens mobilis, et sicut novis gaudet amplexibus, sic notos leviter respuit, citius oblivione contradit.

18 Radulph von Caen, Tancredus. Hrsg. v. Edoardo D’Angelo (CC Cont. Med. 231). Turnhout 2011, hier Kap. 31, S. 10; Wilhelm von Tyrus (Anm. 1), XIV, 6, S. 637. Zu weiteren Rollenbildern vgl. die Beiträge von Christoph Auffarth und Christoph T. Maier in diesem Band.

19 Darunter finden sich bezeichnenderweise mehrere Witwen: Regesta regni Hierosolymitani (MXCVII-MCCXCI). Hrsg. v. Reinhold Röhricht. Innsbruck 1893 (nachfolgend RRH), Nr. 192, 254, 297 (Witwe), 311, 350, 401, 483, 504 (Witwe), 510, 522 (Witwe), 553 (Witwe), 587, 594, 621, 640, 736, 784, 1026 (Witwe), 1058, 1069, 1086, 1372, 178a, 238a, 280a (Witwe), 323a, 331a, 365d, 434a, 594, 787b, 879 (Witwe), 1065, 1065a (Witwe), 1072b (Witwe), 1084a, 1088a (Witwe), 1098b, 1198d, 1271a (Witwe), 1312a (Witwe), 1335b (Witwe), 1349a, 1358b, 1438a (Witwe). 
konnte. ${ }^{20}$ Aber häufig wurden selbst Erbinnen einfach titellos als uxores bezeichnet und ihren Männern nachgestellt. ${ }^{21}$

Abgesehen von den Herrscherinnen der ersten Hälfte des 12. Jahrhunderts, von denen gleich die Rede sein wird, spiegeln die Urkunden vor allem im 13. Jahrhundert (einige wenige) aktive Frauen. Die Ausstellerinnen dieser Diplome bezeichneten sich stets mit den Titeln regina, principessa, comtessa oder domina. Ein Fall nennt sogar eine eigene Hofhaltung (curia). ${ }^{22}$ Die für das 13. Jahrhundert nachweisbare Verwendung eigener Frauensiegel zeugt von einem gesteigerten herrschaftlichen (Selbst-)Bewusstsein, während Königinnensiegel bereits für das 12. Jahrhundert überliefert sind. Dabei ist auffallend, dass die Frauensiegel durchgehend kleiner waren als jene der Könige. ${ }^{23}$

\section{Herrschaft und Regentschaft im 12. Jahrhundert}

Auch wenn Wilhelm von Tyrus weiblichem Herrschaftshandeln grundsätzlich skeptisch gegenüberstand, zeigen seine Ausführungen doch, dass verheiratete und verwitwete Frauen durchaus in wichtigen Positionen agierten und in ihrem

20 Diplome auch im Namen der Gemahlin: RRH (Anm. 19), Nr. 79, 82, 108, 110, 118, 130, 134, 146, 150, 154, 158, 190, 191, 212, 252, 255, 257, 274, 295, 344, 345, 349, 391, 426, 527, 550, 558, 569, 610, 719, 800, 866,978, 1265, 1327, 365e, 434c, 457a, 458a, 530b, 569b, 938a, 1166a; Diplome mit Zustimmung der Gemahlin: RRH Nr. 44, 111, 112, 113, 147, 159, 183, 193, 195, 198, 201, 206, 212, 218, 248, 251, 266, 270, 273, 284, 298, 308, 328, 330, 332, 335, 339, 340, 348, 360, 361, 373, 378, 380, 390, 414, 417, 419, 423, 425, 426, 433, 447, 448, 454, 472, 473, 478, 479, 493, 504, 511, 523, 524, 525, 531, $533,539,542,545,549,551,559,562,583,587,588,590,595,596,605,611,619,620,624,628,629$, $630,647,656,680,689,695,723,742,753,768,800,810,812,845,877,878,904,933,1067,1100$, 1104, 1135, 1175, 1189, 1210, 1217, 1228, 1266, 102a, 183a, 488a, 530a, 535b, 553a, 559c, 608a, 611b, 644a, 651c, 657d, 774a, 859b. Dazu kommen die Bestätigungen der Jerusalemer Königinnen, vgl. Die Urkunden der lateinischen Könige von Jerusalem, 4 Bde. Hrsg. v. Hans Eberhard Mayer (MGH Diplomata Regum Latinorum Hierosolymitanorum). Hannover 2010 (nachfolgend D/DDJerus.).

21 Vgl. etwa RRH (Anm. 19), Nr. 625 (in scharfem Kontrast zu 553), 228, 253, 282, 292, 314, 347, 627, 336a.

22 Agnes von Skandalion: RRH (Anm. 19), Nr. 1384 (evtl. mit angekündigtem Siegel); Alice von Toron und Kerak: RRH Nr. 1056, 1073 (Rechtsgeschäft vor Alice samt curia und ihren Gefolgsleuten); Eschiva von Tiberias: RRH Nr. 522 (mit angekündigtem Siegel), 1336a; Juliane von Caesarea: RRH Nr. 736 (mit angekündigtem Siegel), 768, 810 (mit angekündigtem Siegel), 818-819 (mit angekündigtem Siegel), 866; Margarete von Sidon: RRH Nr. 1215a; Maria von Beirut: RRH Nr. 401; Sibylle von Jerusalem als Gräfin von Jaffa-Askalon: RRH Nr. 553. Dazu kommen die diplomatischen Aktivitäten der Jerusalemer Königinnen, vgl. dazu DJerus. (Anm. 20). Zum Siegelwesen in Outremer vgl. Hans Eberhard Mayer u. Claudia Sode, Die Siegel der lateinischen Könige von Jerusalem (MGH Schriften 66). Wiesbaden 2014.

23 Mayer u. Sode (Anm. 22), S. 10, $21 \mathrm{f}$. 
Tun auch Unterstützung fanden. Konkrete Beispiele für weibliche Erbfolgen werden von den späten 1120er-Jahren an greifbar, als die Konsolidation der Kreuzfahrerherrschaften abgeschlossen war. Herrschte vorher noch eine beträchtliche Fluktuation in den Reihen der Lehnsnehmer, so stabilisierte sich nun die Situation: Die Lehen gingen zunehmend in erblichen Familienbesitz über und die großen Dynastien Outremers begannen sich $\mathrm{zu}$ formieren. ${ }^{24} \mathrm{Im}$ Zuge dieser Entwicklung kam den Frauen eine wichtige Stellung im sozialen Netzwerk des kriegerischen Outremer zu, in dem viele Männer im Kampf starben. Als Trägerinnen von Verwandtschaftsbanden und (ererbten) Herrschaftsansprüchen sorgten sie für dynastische Kontinuität und Rechtssicherheit - beides Aspekte, die für die Ausbildung tragfähiger Familientraditionen entscheidend waren. ${ }^{25}$

Der Jerusalemer Elite dürfte damals zudem ein Erbschaftsfall auf höchster Ebene als Musterbeispiel gedient haben: Der dortige Königsthron war in den ersten rund dreißig Jahren seines Bestehens immer im Kreis der männlichen Verwandten Gottfrieds von Bouillon, der nach der Eroberung der Heiligen Stadt dieses Kreuzfahrerreich gegründet hatte, weitergegeben worden. Als sich abzeichnete, dass dem dritten König, Balduin II., kein männlicher Erbe beschieden sein würde, dafür aber vier Töchter, begann dieser von 1126 an eine ungewohnte Vorsorge zu treffen, um dem Königreich eine starke Führung zu garantieren und die Herrschaft seiner eigenen Dynastie zu wahren. Er begann kurz nach dem Tod seiner Gemahlin, seine älteste Tochter Melisende zur heres regni, zur Erbin des Reiches, zu stilisieren. ${ }^{26}$ Dass dies keineswegs selbstverständlich war und die Zeitgenossen überrascht haben könnte, deutet sich in einem noch kurz zuvor aufgesetzten Vertragswerk mit Venedig an, das von einem männlichen Thronfolger ausgegangen zu sein scheint - obwohl man wusste, dass der König nur vier Töchter hatte. ${ }^{27}$

24 Vgl. Joshua Prawer, Histoire du royaume latin de Jérusalem. Paris 1969, S. 470-474; Hans Eberhard Mayer, Herrschaft und Verwaltung im Kreuzfahrerkönigreich Jerusalem. In: Historische Zeitschrift 261 (1995), S. 695-738, bes. S. 699 f.

25 Es ließe sich umgekehrt auch fragen, inwiefern die Herausbildung des weiblichen Erbrechts die Etablierung starker Familien begünstigt hat.

26 DDJerus. (Anm. 20), Nr. 105, S. 263; 109, 269; 124, 288. Zu Melisendes Anfängen vgl. Mayer (Anm. 4), S. 99; Murray (Anm. 3), S. 138-142.

27 DJerus. (Anm. 20), Nr. 764, S. 1336: Si vero alter ad Ierosolimitanum regnum in regem promovendus advenerit, aut superius ordinatas promissiones, antequam promoveatur, sicut ante dictum est, ipsum firmare faciemus, alioquin ipsum nullo modo ad regnum provehi assentiemus [Hervorhebungen d.V.]. Diese Formulierung steht im Kontrast zu jener eines Vertragswerks von 1187, in dem die Ratifikation durch den König und die Königin festgelegt wurde: DJerus. (Anm. 20), Nr. 769. Vgl. zu den beiden Verträgen Goridis (Anm. 6), S. 241-247. 
Balduins Töchter standen am Anfang einer ersten Phase von Herrscherinnen im Heiligen Land. Alle vier waren selbstbewusste Persönlichkeiten, die weder die Konfrontation mit ihren Ehemännern noch mit der Nobilität scheuten und sich gegenseitig unterstützten. ${ }^{28}$ Ein Grund für dieses Verhalten der Königstöchter könnte in östlichen Vorstellungen von weiblichem Herrschaftshandeln liegen. Ihre Mutter war Armenierin und könnte sie mit entsprechendem Gedankengut in Kontakt gebracht haben. ${ }^{29}$ In jedem Fall gilt Melisende als Paradebeispiel einer selbstständig agierenden Königin - ein Bild, das zweifellos auch von ihrer großen Quellenpräsenz und dem Wissen um die (konstitutionelle) Geschichte des Königreichs Jerusalem herrührt. Als nämlich ihr Gatte, Fulko V. von Anjou, seine Gemahlin und die alteingesessene Nobilität Jerusalems, die ihren Aufstieg Melisendes Vater verdankte, aus einflussreichen Positionen $\mathrm{zu}$ verdrängen suchte, formierte sich Widerstand: Der alte Adel unterstützte Melisende entschieden gegen die Pläne Fulkos und stärkte ihre Stellung. Seit 1135 bekräftigte die Königin konsensgebend jedes Diplom, das der König ausstellte - eine Gepflogenheit, die Wilhelm von Tyrus zu seinem eingangs erwähnten Kommentar über die Verweiblichung Fulkos nötigte, und die später zum Usus wurde, wenn ein angeheirateter König, ein rex consors, eine Urkunde ausstellte. ${ }^{30}$ Dabei ging es der Jerusalemer Nobilität weniger um die Durchsetzung der weiblichen Erbfolge und Melisendes Herrschaftsrecht per se, sondern um die Unterstützung jener Dynastie, von deren Wohl der eigene Einfluss abhing. So ist es auch bezeichnend, dass Melisendes Regierungszeit - wohl gegen deren eigenes Empfinden - oft eher als Regentschaft für ihren minderjährigen Sohn Balduin III. angesehen worden zu sein scheint. ${ }^{31}$ Sogar Wilhelm von Tyrus beschreibt Melisende an prominenter

28 Wilhelm von Tyrus (Anm. 1), XIV, 20, S. 658; XV, 26, S. 709 f.; XVII, 18f., S. 785-787.

29 Zu mächtigen Frauenfiguren im Osten vgl. u. a. Lynda Garland, Byzantine Empresses. Women and Power in Byzantium AD 527-1204. London 1998 sowie den Beitrag von Michael Grünbart in diesem Band.

30 DDJerus. (Anm. 20), Nr. 156-168. Zu den Rechten und Pflichten von männlichen consortes vgl. Goridis (Anm.4). Seinen Niederschlag fand die Konsenspflicht der Erbkönigin auch in der Jerusalemer Rechtstradition, vgl. Le Livre au Roi. Hrsg. v. Myriam Greilsammer (Documents relatifs à l'histoire des croisades 17). Paris 1995, Kap. 4, S. 143 f.

31 Wilhelm von Tyrus (Anm. 1), XVII, 13, S. 778 berichtet, dass Balduin III. geraten worden sei, sich der Vormundschaft seiner Mutter zu entziehen, da es sich aufgrund seiner Volljährigkeit nicht mehr zieme, sich dem Willen einer Frau zu unterwerfen, vgl. dazu auch XVI, 3, S. 717. Auch Bernhard von Clairvaux wird in einem Brief an Melisende recht deutlich, Sancti Bernardi Opera (Anm. 16), Nr. 354: Mortuo rege viro tuo, et parvulo rege adhuc minus idoneo ad portanda negotia regni et ad prosequendum regis officium, oculi omnium in te respiciunt et in te solam universa regni moles inclinata recumbit. Für die Übersetzung vgl. Joan Ferrante, A Letter from Bernard of Clairvaux, Abbot (1143-44); http://epistolae.ccnmtl.columbia.edu/letter/246.html (Zugriff: 11.1.2015; Hervorhebungen d.V.). 
Stelle einmal ausdrücklich als filiorum legitimam agens tutelam und nicht etwa als eigenständige Herrscherin. ${ }^{32}$ Mit der Volljährigkeit Balduins III. geriet ihre Position dann auch unter Druck, bis sich selbst ihre letzten Anhänger von ihr abwandten. ${ }^{33}$

Eine ähnliche Entwicklung lässt sich im Fürstentum Antiochia feststellen, in dem der Adel ebenfalls die einzige Thronerbin unterstützte, um die Machtbestrebungen von Alice, Witwe des Erbfürsten und eine Schwester Melisendes, zu unterbinden. Diese habe versucht, so Wilhelm von Tyrus, auf Kosten ihrer kleinen Tochter, der Thronerbin Konstanze, die Macht in Antiochia an sich zu reißen und zu diesem Zweck mit dem Aufbau einer eigenen Klientel begonnen. Um dies zu verhindern, hätten ihre Gegner klammheimlich die Verheiratung der kleinen Konstanze organisiert. ${ }^{34}$ Dadurch galt Konstanze als volljährig und herrschaftsberechtigt, sodass Alices Ablösung an der Spitze des Fürstentums legitimiert war.

In diese Zeit fällt auch eine weibliche Erbfolge auf seigneuraler Stufe, die die bisherigen Beobachtungen bestätigt. Hans Eberhard MAYER skizzierte den Fall des Balduin von Ramla, der in den späten 1130er-Jahren sein Lehen an sein ältestes Kind, seine Tochter Helvis, übergeben habe. Dass die Herrschaft von Helvis nur von kurzer Dauer war, passt zu den Zweifeln an der Herrschaftsbefähigung von Frauen, die bei Wilhelm von Tyrus greifbar sind. Mit Erreichung der Volljährigkeit löste der jüngere Bruder seine Schwester ab; man könnte deshalb annehmen, dass ihre Regierung lediglich als Regentschaft für den Minderjährigen gedacht war. ${ }^{35}$

Diese frühe Phase war von großer Bedeutung für die weitere Konstituierung der Kreuzfahrerherrschaften. Vor allem die Herrschaft Melisendes muss als wichtiger Schritt im konstitutionellen Denken Outremers gewertet werden. ${ }^{36}$ Sie stärkte nicht nur erstmals das Bewusstsein für die Relevanz der Dynastie, sondern sie bestimmte auch die grundsätzliche Rollenverteilung zwischen der Erbherrin und ihrem consors für die Zukunft: Der Mann agierte zwar als ausführender Herrscher, aber seine Gattin legitimierte - wenigstens formal - stets seine Position und seine Entscheidungen. ${ }^{37}$

32 Wilhelm von Tyrus (Anm. 1), XVII, 13, S. 777.

33 Vgl. zu diesen Ereignissen bes. Mayer (Anm. 4); Murray (Anm. 3), S. 138-144.

34 Wilhelm von Tyrus (Anm. 1), XIV, 9, S. 640 f.; XIV, 20, S. 657-659.

35 Hans Eberhard Mayer, Carving Up Crusaders. The Early Ibelins and Ramlas. In: Benjamin Kedar, Hans Eberhard Mayer u. Raymond Smail (Hgg.), Outremer. Studies in the History of the Crusading Kingdom of Jerusalem Presented to Joshua Prawer. Jerusalem 1982, S. 101-118, hier S. $115 \mathrm{f}$.

36 Vgl. auch Mayer (Anm. 4), S. 111; Murray (Anm. 3), S. 144.

37 Vgl. zur consors-Herrschaft ausführlich Goridis (Anm. 4). 
Dennoch darf dieser Zustand nicht als Bekenntnis zur weiblichen (Allein-) Herrschaft per se verstanden werden. Besonders im 12. Jahrhundert wurden immer wieder Männer den eigentlich besseren weiblichen (Erb-)Ansprüchen vorgezogen. ${ }^{38}$ Und wenn sich einige als Herrscherinnen eigenen Rechts betrachteten oder solches anstrebten, tendierten viele Zeitgenossen und besonders politische Gegner offenbar dazu, sie als bloße Regentinnen für die minderjährigen Erben abzutun. In dieser Funktion hatten sie sich prinzipiell um deren Güter zu kümmern, bis das Erbe angetreten werden konnte.

Idealerweise standen den Regentinnen also Männer - seien es Ehemänner, enge Vertraute oder Mitglieder des Haushalts - zur Seite, die sich der aktiven Ausübung der Regierung widmeten. Gerade im kriegerischen Milieu der Kreuzfahrerherrschaften dürfte dies von großer Wichtigkeit gewesen sein, da sich Frauen in der Regel nicht aktiv an militärischen Kampagnen beteiligten. ${ }^{39}$ Auch wenn ihnen deshalb diese Möglichkeit der Demonstration königlicher Handlungsmacht nicht gegeben war, konnten sie sich durch loyale Gefolgsleute als Oberkommandierende betätigen - wie zum Beispiel Melisende, die ihren Vertrauten Manasses von Hierges nicht umsonst ausgerechnet zum Konstabler und damit zum Befehlshaber des Heeres gemacht hatte. Damit unterschieden sie sich im Prinzip nicht von Herrschern, die ebenfalls Vertraute in wichtige Positionen beförderten. Doch waren Herrscherinnen bei der aktiven Ausübung königlicher Macht - gerade im dafür wichtigen militärischen Bereich - weit stärker auf loyale Gefolgsleute beziehungsweise im Idealfall auf ihre Gatten angewiesen. ${ }^{40}$ So war Konstanzes Nachfolge als Fürstin in Antiochia an ihre Heirat mit Raimund von Poitiers gekoppelt, ein Ereignis, das gleichzeitig das Ende ihrer Unmündigkeit markierte. Ohne diesen Schritt hätte sie ihr Erbe nicht antreten können. Diese Verknüpfung von Hochzeit und Erbschaftsantritt wurde später im ,Livre au roi' sogar kodifiziert, sodass die Existenz eines consors die Handlungsmöglichkeiten einer jungen Erbin grundlegend bestimmte, aber auch erweiterte. ${ }^{41}$

Bezeichnend und für die gesamte Entwicklung nicht unbedeutend ist aber die Tatsache, dass es praktisch allen eigenständig agierenden Fürstinnen gelungen ist, nach dem Tod ihrer Ehemänner zumindest zeitweise eine Wiederverheiratung $\mathrm{zu}$ vermeiden und dadurch ihren Handlungsspielraum zu erweitern. Selbst für Melisende scheint der von Wilhelm von Tyrus angedeutete Rollentausch erst nach

38 Vgl. ebd., S. $168 \mathrm{f}$.

39 Vgl. dazu Murray (Anm. 3), S. 134-136 mit weiterführender Literatur.

40 Vgl. ebd.

41 Livre au Roi (Anm. 30), Kap. 6, S. 149. Vgl. auch Goridis (Anm. 4), S. 176-179, dort auch weitere Literatur. 
dem Ableben ihres Gatten und den damit verbundenen Freiheiten nachhaltig zum Tragen gekommen zu sein. Und genau in diesem Punkt - der unmittelbar fehlenden Eheverbindung - unterschied sich diese frühe Gruppe von Fürstinnen von den meisten ihrer späteren Standesgenossinnen, die nicht so unabhängig wirkten. Erst die Witwenschaft sorgte für die Wahrnehmung als eigenständige Herrscherinnen.

\section{Entwicklungen bis ins 13. Jahrhundert}

Eine entscheidende Veränderung brachten die Jahre zwischen 1186 und 1205, die eine zweite Phase ausgeprägter Herrschaftspräsenz von Frauen in Outremer einläutete. Diese Zeit wurde in Jerusalem von einem Thronstreit zwischen den insgesamt fünf Ehemännern der beiden Thronerbinnen und Halbschwestern Sibylle und Isabella geprägt. In dieser Auseinandersetzung versuchten Männer die Krone zu ergreifen, die ihre Rechte explizit über ihre Verbindung mit den Erbinnen herleiteten. Sibylle und Isabella gerieten dadurch in den Brennpunkt der politischen und juristischen Aufmerksamkeit der Nobilität Outremers. Dies erweiterte zwar nicht die Handlungsmöglichkeiten der beiden Protagonistinnen, die den Quellen zufolge hauptsächlich als Überträgerinnen der Herrschaftsrechte wahrgenommen wurden - eine Rolle, die auch ihren Nachfolgerinnen Maria la Marquise und Isabella II. zukam. Aber die Funktion der beiden Erbtöchter sowie die Bedeutung der Dynastie wurden im zeitgenössischen Diskurs dermaßen stark thematisiert, dass neue erbrechtliche Standards geschaffen wurden.

Danach konnten Erbinnen nicht mehr ignoriert werden wie noch im 12. Jahrhundert. Ihre Bedeutung für die Herrschaft wurde nun im ,Livre au roi‘ erstmals explizit ausformuliert, einer Gesetzessammlung, die König Aimerich, vierter Ehemann Isabellas, in Auftrag gegeben hatte. Ziel des Königs war nicht nur die Klärung der Verfassungslage, sondern auch seine eigene Absicherung als consors. Auf diese Weise wurde die Rollenerwartung an die Erbkönigin und ihre Familie erstmals ausdrücklich artikuliert und im konstitutionellen Gedächtnis Outremers verankert. ${ }^{42}$

Auch wenn eine solche Institutionalisierung weiblicher Herrschaftsberechtigung in den Territorien Europas kaum zu beobachten war, ${ }^{43}$ sollte Outremer nicht

42 Vgl. Goridis (Anm. 4), S. 170-176 zum Thronstreit und S. 176-179 zum ,Livre au roi'.

43 Auf eine interessante Ausnahme aus der Champagne hat mich dankenswerterweise Melanie Panse aufmerksam gemacht. Im Jahr 1212 erließ Gräfin Blanca eine Anordnung, die eine weibliche Erbfolge für Burgen und andere befestigte Plätze im Falle fehlender männlicher Erben ausdrück- 
als exzeptionell betrachtet werden. Abgesehen von den herausragenden Frauengestalten um Melisende in der ersten Hälfte des 12. Jahrhunderts scheint die dortige Situation große Ähnlichkeiten zu jener in anderen europäischen Regionen aufgewiesen $\mathrm{zu}$ haben. Besonders im französischen Raum finden sich seit der zweiten Hälfte des 12. Jahrhunderts vermehrt Frauen in Herrschaftspositionen vermutlich aufgrund der Kreuzzüge und der damit verbundenen Abwesenheit vieler Männer. ${ }^{44}$ Da gerade Frankreich eines der wichtigsten Zuwanderungsgebiete Outremers war, könnte es hier zu einem wechselseitigen Wissenstransfer bezüglich weiblicher Herrschaft gekommen sein - eine These, die der weiteren Untersuchung bedarf.

Bezeichnenderweise weist das Heilige Land eine demografisch prinzipiell sehr ähnliche Zusammensetzung wie Frankreich auf. In beiden Regionen lässt sich die Tendenz feststellen, dass die zahlreichen Kriege einen Mangel an männlichen Herrschaftsträgern bedingten. In Outremer spitzte sich diese Situation Ende des 12. Jahrhunderts dramatisch zu, als bei der aus christlicher Sicht katastrophalen Niederlage bei den Hörnern von Hattin im Jahr 1187 das gesamte christliche Heer ausgelöscht oder von Saladin gefangen genommen wurde. Eine Folge davon war, dass zahlreiche Besitztümer plötzlich unter der Verwaltung der zurückgebliebenen Frauen standen, denen mithin nicht nur die Verantwortung für die Lehen und die verbleibenden Familienmitglieder, sondern häufig auch für die Befreiung ihrer Männer und Söhne zukam.

Gerade im Falle einer Kriegsgefangenschaft bei den Muslimen waren diese Aufgaben äußerst komplex: Die Gattinnen und Mütter - erneut betonen die Quellen die maßgebliche Unterstützung durch ihre militärischen Berater oder Lehnsherren - kümmerten sich nicht nur um die Verteidigung ihrer Güter, sondern sie organisierten auch die Lösegeldzahlungen und begaben sich vereinzelt sogar selbst als Geiseln in muslimische Hände, damit sich die Gefangenen eigenhändig um ihre Auslösung bemühen konnten. ${ }^{45}$ Ein Beispiel dafür ist Stefanie von Milly, Erbin der bedeutenden Seigneurie Oultrejourdain. Nach der Hinrichtung ihres Mannes Rainald von Châtillon und der Festnahme ihres Sohnes durch Saladin war sie für die Rolle der Regentin prädestiniert. Tatsächlich weisen ihr

lich gestattete: The Cartulary of Countess Blanche of Champagne. Hrsg. v. Theodore Evergates. Toronto u. a. 2010, Nr. 450, S. $407 \mathrm{f}$.

44 Vgl. Melanie Panse in diesem Heft zur Situation in der Champagne sowie Anm. 43 oben. Allgemein zur Herrschafts- und Machtentfaltung von Frauen in Europa vgl. Patrick Corbet, Entre Aliénor d'Aquitaine et Blanche de Castille. Les princesses au pouvoir dans la France de l'Est. In: Zey (Anm. 3), S. 225-244.

$45 \mathrm{Zu}$ Gefangenschaft im Heiligen Land vgl. Goridis (Anm. 6), zur Rolle von Frauen bes. S. 293295, 339-341. 
alle Quellen eine zentrale Rolle zu. Sie verhandelte höchstpersönlich mit Saladin, und dem muslimischen Autor Abu Shama zufolge sei ihre Autorität bei ihren um sie versammelten Gefolgsleuten unbestritten gewesen. ${ }^{46}$

Auf jeden Fall sorgte die einschneidende Erfahrung von Hattin zusammen mit den Ergebnissen des Jerusalemer Thronstreits im 13. Jahrhundert für eine gesteigerte Wertschätzung weiblichen Herrschaftshandelns. Das schlug sich in der Akzeptanz herrschender Frauen nieder, die sich, wie beschrieben, anhand der urkundlichen Überlieferung nachvollziehen lässt.

Ähnliches gilt für bedeutende, von der Haute Cour abgesegnete Regentschaften, in denen sich die Position von Frauen bis in die Mitte des 13. Jahrhunderts konsolidierte. Ausgehend von den Errungenschaften des ,Livre au roi', der die Regentschaftsrechte der thronvererbenden Familienlinie einschließlich der Erbinnen forcierte, wurde die Regierungsbefähigung beider Geschlechter schrittweise in der rechtlichen und politischen Vorstellungswelt Jerusalems verankert. ${ }^{47}$ Als Höhepunkt dieser Entwicklung ist zweifellos das berühmte Rechtsbuch Johanns von Ibelin anzusehen. Es verfügt, dass in Abwesenheit des Königs und ausdrücklich der Königin die Regentschaft dem oder der nächsten Verwandten (le plus prochein, seit home ou feme) gebühre, bis die Kinder volljährig seien oder persönlich ins Königreich kämen, um die Herrschaft zu übernehmen. Selbst die Rechte angeheirateter Königinnen auf die Regentschaft wurden speziell hervorgehoben. ${ }^{48}$ Die Zeit der 1240er- bis 1260er-Jahre, bis erstmals wieder ein volljähriger Thronerbe zur Verfügung stand, kann denn auch - gleichsam als eine dritte Phase weiblicher Herrschaftspräsenz - als Epoche der Regentinnen in Jerusalem be-

46 Chronique d'Ernoul et de Bernard le Trésorier publiée pour la première fois, d'après les manuscrits de Bruxelles, de Paris et de Berne, avec un essai de classification des continuateurs de Guillaume de Tyr. Hrsg. v. Louis De Mas Latrie. Paris 1871, Kap. 21, S. 253; Eracles (Anm. 17), XXIV, 20, S. 131; Abu Shama, Le livre des deux jardins. Histoire des deux règnes. Celui de Nour ed-Dîn et celui de Salah ed-Dîn (Recueil des Historiens des Croisades, Historiens Orientaux 4). Paris 1898, S. 1-525, hier S. 332f. Vgl. auch Goridis (Anm. 6), S. 294.

47 Hier sind v. a. die jahrzehntelangen Bemühungen Alices von Champagne-Jerusalem um den Jerusalemer Regentenposten zu nennen, vgl. Goridis (Anm. 4), S. 181-183, 189, $190 \mathrm{f}$. Zu den entscheidenden politischen Entwicklungen der 1240er-Jahre vgl. zudem David Jacoby, The Kingdom of Jerusalem and the Collapse of Hohenstaufen Power in the Levant. In: Dumbarton Oaks Papers 40 (1986), S. 83-101, hier S. 88-91.

48 Johann von Ibelin, Le Livre des Assises. Hrsg. v. Peter Edbury (The Medieval Mediterranean. Peoples, Economies and Cultures, 400-1500 50). Leiden u.a. 2003, S. 805. Zur Rolle von Frauen bei Johann von Ibelin vgl. zudem Peter Edbury, Women and the Customs of the High Court of Jerusalem According to John of Ibelin. In: Damien Coulon u.a. (Hgg.), Chemins d'Outre-Mer. Études d'histoire de la Méditerranée médiévale offertes à Michel Balard. Paris 2004, S. 285-292. 
zeichnet werden, in der immer wieder Frauen an die Spitze des Reiches gestellt wurden. ${ }^{49}$

\section{Frauen und Männer in Outremer - ein Fazit}

Frauen spielten für das politische Handeln in Outremer spätestens seit den 1130er-Jahren eine wichtige Rolle. Die Idee der weiblichen Erbfolge war bereits früh verankert und notwendig, nicht zuletzt weil die ständige Kriegsbereitschaft ihren Tribut an Männern forderte. Zweifellos waren Herrschaftshandeln und -nachfolge männlich dominiert; Ehefrauen, Witwen und Mütter waren vor allem dann zugelassen, wenn keine Alternative denkbar war. So betrachteten Geschichtsschreiber weibliche Herrschaft häufig mit Skepsis. Frauen, die Regierungsaufgaben übernahmen, wurden eher als verwandtschaftlich legitimierte Regentinnen auf Zeit gesehen denn als Herrscherinnen eigenen Rechts. Sie sollten zügig von Männern abgelöst oder, so der Tenor der Chronisten, zumindest tatkräftig von diesen unterstützt werden.

Große Bedeutung hatten Frauen aber für die Herrschaftslegitimation. Gerade Erbinnen kam schon früh die Aufgabe zu, einerseits die Ansprüche ihrer Männer auf ihr Erbgut zu bekräftigen und andererseits deren aktives Herrschaftshandeln, das formal der Zustimmung der Erbin bedurfte, abzusichern. Diese geschlechterspezifische Rollenverteilung zwischen Erbin und consors festigte sich gegen Ende des 12. Jahrhunderts im Jerusalemer Thronstreit und nach der Schlacht von Hattin, bis sie in die schriftlich fixierte Jerusalemer Rechtstradition einging.

In der Folge erweiterte sich das politische Tätigkeitsspektrum von Frauen, auch wenn die potentiell möglichen Herrschaftsfunktionen keineswegs immer alle ausgeübt werden mussten. Obwohl Männer die politische Landschaft Outremers nach wie vor bestimmten, konnte gerade der legitimatorische Aspekt nicht mehr einfach übergangen werden und nach dem Ableben der Erbtochter sogar zu Schwierigkeiten für ihren Witwer führen. ${ }^{50}$ Die Erbinnen waren dadurch zu einem zentralen Element des fränkischen Herrschaftssystems aufgewertet. Ohne Erbherrscherin war ein außerdynastischer Herrscher nicht denkbar - wobei aber auch umgekehrt eine Erbin auf einen Gatten angewiesen war, um als Herrscherin agieren zu können und akzeptiert zu werden. Nicht selten war ihr Herrschaftsantritt an eine Vermählung gebunden.

49 Vgl. Murray (Anm. 3), S. 157f.; Peter Edbury, John of Ibelin and the Kingdom of Jerusalem. Woodbridge 1997, S. 58f., 78-93, als Vergleich zur Situation in Jerusalem dort auch Überlegungen zur Rolle der Plaisance von Antiochia als Königin von Zypern.

50 Vgl. dazu Goridis (Anm. 4). 
Speziell großen Handlungsspielraum scheinen Witwen genossen $\mathrm{zu}$ haben, besonders wenn sie bereits über eigene Nachkommen verfügten und sich, wie Melisende sowie Alice und Konstanze von Antiochia, nachhaltig gegen eine Wiederverheiratung sperren konnten. Diese Fürstinnen verschwanden nicht mehr im Schatten eines Ehemanns - wie die meisten Gemahlinnen - und agierten als eigenständige Herrscherinnen, die männliche Vertraute gezielt mit jenen Posten betrauten, die sie als Frauen nur schwer ausfüllen konnten. Auch Mütter von Potentaten, die gemäß den Quellen öfters in deren Beraterkreis eingebunden waren, scheinen mitunter beträchtlichen Einfluss auf ihre Söhne ausgeübt zu haben. In der Regel geben die Quellen nur zurückhaltend Einblick in diese Aktivitäten.

Die folgende Zusammenstellung deutet allerdings an, dass Frauen, erbberechtigt oder nicht, als Ehefrauen, Mütter und Witwen stark auf die männerdominierte Politik einwirken konnten: Sie engagierten sich in Hochzeitsvorbereitungen und Eheverhandlungen, die das sozio-politische Gefüge Outremers bestimmten. ${ }^{51}$ Sie verhandelten mit anderen Herrscherinnen, was auf eine weibliche Ebene der Diplomatie hinweist. ${ }^{52}$ Zudem nahmen sie mitunter beträchtlichen Einfluss: sei es auf ihre Männer und Söhne, ${ }^{53}$ auf ihre Töchter und Nichten, deren Erziehung ihnen oblag, ${ }^{54}$ oder gar auf die Besetzung von Führungspositionen wie die des Patriarchen oder des Regenten für ihre unmündigen Kinder. ${ }^{55}$ So stand Melisende auch nach ihrem Rückzug aus dem aktiven Königinnengeschäft ihrem Sohn mit Rat und Tat zur Seite, und Agnes von Courtenay, die Mutter König Balduins IV., war beim Abschluss von Regierungsgeschäften anwesend, die sie angeblich penitus hortans begleitete. ${ }^{56}$

Während der Abwesenheit ihrer Männer übernahmen Frauen einzelne Herrschaftsfunktionen wie die Verwaltung von Städten, die Aussendung diplomati-

51 Wilhelm von Tyrus (Anm. 1), XVIII, 31, S. 856f.; Eracles (Anm. 17), XXV, 11, S. 151f.; DJerus. (Anm. 20), Nr. 645.

52 Wilhelm von Tyrus (Anm. 1), XVII, 19, S. 786 f.; Eracles (Anm. 17), XXV, 11, S. $151 \mathrm{f}$.

53 Wilhelm von Tyrus (Anm. 1), XVII, 30, S. 804; XXII, 26, S. 1049; XXII, 29, S. 1058; Eracles (Anm. 17), XXV, 11, S. 151f.; XXXIV, 19f., S. 466 f.; siehe auch die Ausführungen oben zu Melisende.

54 Wilhelm von Tyrus (Anm. 1), XVIII, 31, S. 856f.; XXI, 2, S. 962; XXI, 15, S. 980 f.

55 Ebd., XVIII, 20, S. 840; Eracles (Anm. 17), XXIII, 38f., S. 58-60; XXX, 11, S. 305; Philipp von Novara, Guerra di Federico II in Oriente (1223-1242). Hrsg. v. Silvio Melani (Nuovo Medioevo 46). Napoli 1994, Kap. 19, S. 74; Cronaca del Templare di Tiro (1243-1314). La Caduta degli Stati Crociati nel racconto di un testimone oculare. Hrsg. v. Laura Minervini. Neapel 2000, Kap. 149, S. 142.

56 Wilhelm von Tyrus (Anm. 1), XVII, 30, S. 804; XXII, 30, S. 1058. 
scher Missionen oder gar die Organisation militärischer Aktivitäten. ${ }^{57}$ Gerade im Falle gefangenschaftsbedingter Abwesenheit von Gatte oder Sohn wurden ihre Herrschaftsrechte ausdrücklich geschützt, sodass vorhandene Ressourcen weiter genutzt werden konnten. ${ }^{58}$ Zudem muss bedacht werden, dass gerade hochgestellte Frauen oft über große Heiratsgüter und Wittümer verfügten, die ihnen nicht nur eine gewisse Verhandlungsmacht verschafften, sondern aus denen auch beträchtliche Finanzmittel erwuchsen. ${ }^{59}$ Und schließlich dürfen auch die stifterischen Aktivitäten von Frauen nicht vergessen werden, die, so Nikolas JASPERT, zu den „Varianten indirekten politischen Handelns“ gehören, die in Quellen häufig nicht so prominent behandelt wurden, für die öffentliche Wahrnehmung einer Herrscherin aber von großer Bedeutung waren. ${ }^{60}$

Die Geschichtsschreiber modifizierten auch ihre genderspezifischen $\mathrm{Zu}$ schreibungen an das Herrscherpaar: Übten sich Frauen für längere Zeit in üblicherweise Männern zugeschriebenen Tätigkeiten, wurden sie gerade im 12. Jahrhundert dafür entweder kritisiert oder virilisiert. Umgekehrt wurden Männer, die - wie Fulko - nicht in der Lage waren, ihren Herrscherpflichten nachzukommen, effeminiert. Auf subtilere, aber genauso eindrückliche Weise rückte Juliane von Caesarea ihre Ansprüche ins rechte Licht und demonstriert damit, dass sich seit dem ausgehenden 12. Jahrhundert das überkommene, gewohnheitsrechtliche Rollenbild der Frau in Outremer gewandelt hatte: Sie zelebrierte ganz wörtlich genommen einen Rollentausch, indem sie in ihren Urkunden die sonst übliche Zustimmung der Gattin mit der des Herrn vertauschte: Juliana, domina Caesareae, [...] assensu mariti Ademari [...]. ${ }^{61}$

57 Fulcher von Chartres, Fulcheri Carnotensis historia Hierosolymitana (1095-1127). Hrsg. v. Heinrich Hagenmeyer. Heidelberg 1913, II, 14, 2-5, S. 421-423; Wilhelm von Tyrus (Anm. 1), X, 17, S. 474; XIV, 6, S. 637 f.; Eracles (Anm. 17), XXIII, 55, S. 68; Itinerarium peregrinorum et gesta regis Ricardi. Chronicles and Memorials of the Reign of Richard I. Hrsg. v. William Stubbs (Rerum Britannicarum medii aevi scriptores 38 [Rolls Series]). London 1884, V, 35, S. 348.

58 Goridis (Anm. 6), S. 196-198.

59 Wilhelm von Tyrus (Anm. 1), XVIII, 22, S. 843.

60 Vgl. Nikolas Jaspert, Indirekte und direkte Macht iberischer Königinnen im Mittelalter. „Reginale“ Herrschaft, Verwaltung und Frömmigkeit. In: Zey (Anm. 3), S. 73-130, hier S. 110-123, zit. S. 112, für allgemeine Überlegungen dazu; vgl. auch Jaroslav Folda, Melisende of Jerusalem. Queen and Patron of Art and Architecture in the Crusader Kingdom. In: Therese Martin (Hg.), Reassessing the Roles of Women as ,Makers‘ of Medieval Art and Architecture. Bd. 1 (Visualising the Middle Ages 7:1). Leiden 2012, S. 429-477; Helen A. Gaudette, The Spending Power of a Crusader Queen. Melisende of Jerusalem. In: Theresa Earenfight (Hg.), Women and Wealth in Late Medieval Europe. New York 2010, S. 135-148.

61 RRH (Anm. 19), Nr. 736 mit einer Urkunde aus dem Jahr 1197. 
Auch wenn Juliane damit eine Ausnahme blieb und politische Entscheidungen, wie insbesondere die diplomatische Überlieferung verdeutlicht, in der Regel von Männern dominiert wurden, zeigt ihr Beispiel das Handlungspotential einer Erbin auf. Daneben verfügten Frauen über ein breites Spektrum an subtileren Formen von Herrschaftsfunktionen, das die Chronistik häufig nur ungenügend zur Kenntnis nahm. Doch steht fest, dass Frauen vielfältige Handlungsmöglichkeiten und Funktionen besaßen, die jenen männlicher Herrschaftsträger nicht nachstanden. Vielmehr bedingten und ergänzten sich diese gegenseitig, sodass das Funktionieren des sozio-politischen Gefüges Outremers nur vor dem Hintergrund dieses Zusammenspiels seiner Herrscherinnen und Herrscher verstanden werden kann. 